\title{
Protée
}

\section{Origine, provenance et surgissement. La recherche de la cause originale dans le platonisme grec}

\section{Georges Leroux}

Volume 28, numéro 1, 2000

Variations sur l'origine

URI : https://id.erudit.org/iderudit/030579ar

DOI : https://doi.org/10.7202/030579ar

Aller au sommaire du numéro

Éditeur(s)

Département des arts et lettres - Université du Québec à Chicoutimi

ISSN

0300-3523 (imprimé)

1708-2307 (numérique)

Découvrir la revue

Citer cet article

Leroux, G. (2000). Origine, provenance et surgissement. La recherche de la cause originale dans le platonisme grec. Protée, 28(1), 7-18.

https://doi.org/10.7202/030579ar
Résumé de l'article

La conception philosophique grecque de l'origine s'amorce avec la recherche présocratique des causes premières et se développe dans la métaphysique de Platon. La doctrine platonicienne intègre les éléments narratifs de la physique et des cosmogonies antérieures, mais elle entreprend de les transformer par la position d'une origine du monde qui soit hors du temps. Cette étude examine plusieurs aspects de cette conception, en particulier dans le texte du Timée et dans le néoplatonisme postérieur. 


\section{O RIG IN E, PRO VEN AN CE ET SU RG ISSEM EN T LA RECHERCHE DE LA CAUSE O RIG IN AIRE DANS LE PLATO NISME GREC}

La résonance de l'origine envahit le champ entier de la pensée grecque, mais dans le moment même où cette pensée s'exerce, de toutes ses forces, à tendre les dimensions qui pourraient capter l'origine dans le temps, elle travaille également dans une autre direction, soutenue par un effort qui est celui de la métaphysique, à déporter hors du temps la puissance de cette origine. Elle parvient ainsi, et principalement dans le platonisme, à constituer comme origine du temps une cause exemplaire qui en est radicalement séparée. Cette double portée de la pensée grecque a captivé tous les interprètes, et notamment Hegel, qui y ont vu le signe d'une culture obsédée par la force corruptrice du temps et profondément désireuse de parvenir à la réconciliation que seules autorisent la position du déterminisme et la pensée du temps cyclique. Une perfection atemporelle peut-elle sauver le temps du désastre qui le mine en son principe? C'est la tentative extrême de la métaphysique grecque. On parvient ainsi à une représentation du devenir qui échappe aux apories de l'origine, puisque le devenir n'est pas le déroulement d'un processus dont l'esprit pourrait entreprendre de retracer le point de départ, mais la répétition insondable des effets éloignés et imparfaits d'une cause séparée et parfaite. Une origine qui ne serait qu'un point de départ serait liée au processus qu'elle déclenche et elle en ferait partie, alors qu'il est question de poser une origine transcendante qui soit en même temps provenance et surgissement. Car l'éternité du monde, qui constitue pour la pensée grecque une position de dépassement, n'implique pas qu'il soit privé d'origine. Le platonisme représente à lui seul l'exemple d'un monde de pensée où ces deux efforts sont développés de manière conjointe, puisqu'on y trouve aussi bien le désir de l'origine et le projet de la retrouver en s'y reliant par la philosophie que l'affirmation de l'absolue séparation et de la distance qui infiniment détache de ce qui est originaire. Ce double mouvement structure déjà le texte de Platon, mais il ne s'accomplit de manière synthétique et véritablement réconciliée - quelques siècles plus tard - que chez Plotin et à partir de lui chez Proclus et chez Damascius. Les mythes de chute 
et de retour, repris de l'orphisme et du pythagorisme, contribuent à en nourrir la représentation, mais pour l'essentiel, dans la tradition platonicienne, ce mouvement trouve sa substance dans la philosophie.

Le langage requis pour exprimer ce mouvement ne pouvait pas ne pas se nourrir de ces métaphores indépassables de la source et du surgissement. Pourquoi indépassables? Parce que ces métaphores se trouvent privées dès le point de départ d'une interprétation neutre, qui constituerait leur portée métaphysique propre. Comme les images de la lumière et de la clarté, elles ne possèdent aucun endeçà purement métaphysique qui, agissant comme leur sens propre, condamnerait leur expression figurée au statut d'un registre primitif et interprétable. La limite du langage de la métaphysique se tient ici, sur le seuil de ces expressions de l'origine qui présentent le temps dans sa manifestation pure. Le rapport à l'origine est d'ores et déjà celui d'une provenance et d'un surgissement, et de manière tout à fait significative, contrairement aux langues qui en dérivent, la langue grecque n'investira pas fortement dans son lexique ce rapport métaphysique. On pense ici, par comparaison, à la richesse de la langue allemande, qui concentrera dans une myriade de composés la pensée de ce qui est -ur, la pensée de ce qui surgit en provenant, saisi dans le seul mouvement de cette provenance. Que va choisir la langue philosophique grecque pour tenter de saisir l'originarité? On peut isoler deux registres: le premier, qui se structure déjà dans la pensée présocratique, est celui de l'arkhè, un terme dont la sédimentation dans la langue poétique est d'une richesse considérable et qui va conduire, dès Platon, à la conceptualisation de la causalité; le second est la purification du langage de la provenance et du surgissement, dans un surinvestissement abstrait du langage prépositionnel du génitif. Les prépositions ek et apo, dont la complémentarité se montre justement dans la structure du cas qu'elles commandent, vont devenir, dans le moyen et dans le néoplatonisme, le vecteur principal de la métaphysique de l'origine, à laquelle elles fourniront le langage d'une dépendance pure, isolée par l'absence de termes, de toute référence temporelle, de toute histoire. Ainsi pourra s'instaurer une pensée du principe et de l'arkhè, purifiée de toute signification entachée par la concrétude d'une origine dans le temps. Le paradoxe de l'origine grecque trouve dans ce langage extrême son expression la plus parfaite: l'origine, recherchée pour penser le temps, n'est pensable que dans une dépendance qui sacrifie le temps et qui ouvre sur une altérité absolue, que les penseurs néoplatoniciens penseront comme différence pure. Dans l'exposé qui suit, je propose de reparcourir ces deux registres, dans le but d'en montrer la complémentarité: aucune pensée de la cause ne peut se passer d'une pensée de la différence, et si l'origine leur est commune, il faut tenter de voir ce qui dans la causalité est pensée de la dépendance comme provenance et surgissement, mais dans ce moment même, saisir également comment cette dépendance est l'expression de la différence pure.

\section{L'ARKHÈ PREMIĖRE}

L'histoire de l'arkhè montre un concept d'une ample équivocité, ouvert aussi bien sur les significations de la cause originaire que sur les éléments de la physique qui déterminent la nature. S'agit-il de principes originaires, s'agit-il de matières élémentaires et ultimes? Il semble impossible d'éclairer le sens de l'arkhè sans faire intervenir le concept de la phusis, en voie d'être saisie comme nature et comme être dans la pensée des présocratiques. Mais ce chemin est déjà bien balisé, il a été l'objet de travaux admirables et son sens apparaît plutôt dans sa destination que dans son point de départ ${ }^{1}$. Le point de départ est la recherche de l'élément primitif et déterminant, saisi comme cause, chez les présocratiques et la destination est son aboutissement dans la métaphysique de Platon, qui se réapproprie l'ensemble des significations présocratiques pour les articuler dans une métaphysique de la causalité. Platon fait lui-même, dans le Phédon, le récit de ce développement et sa critique de la pensée présocratique suppose acquise la position d'une cause transcendante. Les aspects de 
cette métaphysique qui appartiennent encore au récit du Peri phuseôs ancien sont certes nombreux et ils paraissent indissociables de l'effort pour penser l'arkhè hors du temps ${ }^{2}$. Il y a toujours en effet, sur l'horizon de la métaphysique, une tentative de penser un «au commencement", dont l'ultime expression se retrouve dans le Timée. C'est là que nous devons la reprendre, car c'est dans le Timée que l'origine comme cause est présentée par Platon dans un discours qui accepte le discours vraisemblable du mythe ancien, c'est-à-dire de la cosmologie d'Hésiode et des présocratiques, mais qui cherche à en transcrire la physique sur un registre supérieur. La synthèse entre la narration de l'origine et la métaphysique intemporelle atteint dans ce dialogue son point de tension ultime.

Le projet de dire ce qui s'est passé la première fois, ce qui a été non seulement le premier événement, mais l'événement même du monde en tant que phusis, est en effet déjà au foyer de l'épopée et des généalogies hésiodiques. La Théogonie doit raconter comment les dieux naquirent et cette naissance est elle-même, en son principe, la métaphore de toute l'entreprise de la pensée $^{3}$, dans la mesure où la pensée ne saurait se proposer à elle-même un objet plus élevé et plus sublime que le récit de l'origine de l'être souverain et éternel. Le surgissement des discordes primitives est à cet égard aussi essentiel au récit que la constitution même de l'être souverain et ses généalogies archaïques. On ne le voit nulle part aussi bien que dans la réutilisation des récits de généalogie et de conflit qui vont servir de matrice aux enquêtes de la première physique. Empédocle et Anaximandre, pour ne mentionner que deux des textes les plus importants, versent naturellement dans le langage épique la saisie de cette arkhè originaire, dans laquelle ils voient d'abord une genèse. L'identification chez Anaximandre de l'origine et de l'infini est certes la détermination la plus ancienne de cette arkhè dans la pensée grecque, et son importance vient de la pensée de l'origine comme source éternelle des êtres: le «ce dont provient» est consubstantiel à l'être qu'il engendre ${ }^{4}$. C'est Simplicius qui nous apprend qu'Anaximandre fut le premier à nommer le substrat arkhè, une désignation qu'il hérite selon toute vraisemblance du récit de l'épopée; c'est lui aussi qui nous livre le texte de ce fragment crucial:

Anaximandre [...] a dit que l'arkhè et l'élément des êtres est l'infini. (DK, 12 A 9)

Anaximandre [...] dit que l'origine des êtres est l'infini, car de lui toutes choses naissent et en lui toutes choses se résolvent.

$(\mathrm{DK}, 12 \mathrm{~A} 14)^{5}$

C'est à cette généalogie de l'être que Platon fait écho quand, dans le Timée (27c), il propose cette invocation préalable au festin de la connaissance antique: la recherche de l'arkhè s'effectue sous le regard bienveillant des dieux, alors que le langage de la philosophie entreprend une explication de l'origine dont ils sont eux-mêmes les principes tutélaires. L'arkhè appartient en effet toujours déjà à cet autre monde, qui ne participe pas à la genèse, qui n'en subit d'aucune manière la détérioration et qui, pour des raisons que la prière philosophique peut chercher à conjurer, est néanmoins présent dans la pensée de ce monde et donc dans ce monde lui-même. Cette césure était-elle active dans la pensée des présocratiques? On peut en discuter longuement, surtout si on fait intervenir la matrice orientale de leur pensée, c'est-àdire les généalogies du mythe mésopotamien. Il faut aussi évoquer sans doute les éléments qui, de l'orphisme autant que du pythagorisme, ont contribué à poser la notion d'une différence ontologique des deux mondes. Dans ces pensées, dont le mythe appartient à un projet existentiel de purification, c'est d'abord l'origine de l'âme qui est recherchée, et non pas l'origine de la matière et du kosmos, c'est-à-dire de la phusis comme totalité. Le mythe de la chute est à proportion, dans ces traditions complexes, plus déterminant que tout le récit de la genèse des entités nocturnes et des cycles cosmiques.

La séparation de l'origine, quand on la replace sur cet horizon des récits de genèse, est le problème cardinal de la pensée grecque: le khôrismos platonicien n'en constitue que la figure la plus évidente et la plus austère, puisque la séparation chez Platon est définitive, réelle, insurmontable. Même quand elle 
sera interprétée comme négativité pure, c'est-à-dire comme manque et absence de l'origine, cette séparation se placera comme un abîme infranchissable. Il est important de montrer cependant que dans le texte platonicien elle s'intègre dans une entreprise philosophique qui n'est pas que négative. Le Timée, de par le dispositif ambivalent du récit vraisemblable qu'il met en place, tente en effet l'impossible: l'articulation du monde visible et de l'histoire humaine, assujettis au trouble et à la corruption de tout ce qui est engendré, avec la cause originaire, placée comme modèle éternel. La séparation peut-elle être vaincue? Le mécanisme du monde, son appareil structuré de causes, n'est pas réellement coupé de son modèle, et tout le risque de cet extraordinaire récit est d'arriver à investir la figure du Démiurge pour lui faire jouer le rôle du passeur de l'origine éternelle dans le temps ${ }^{6}$. Le modèle de l'appareil filtrant des causes en est l'artefact conceptuel et sa physique du reflet troublant n'interdit pas de penser un passage, et à tout le moins des traces. L'éternel investit le temps, y laisse des vestiges dans un traçage originaire mystérieux que Platon représente dans la description du crible et des faisceaux. C'est parce que le récit philosophique accepte la dimension narrative qu'il peut se saisir du concept présocratique de l'arkhè dans sa dimension de commencement, de déploiement de la genèse de la phusis, mais c'est aussi parce qu'il en soumet l'interprétation au prisme du mythe démiurgique qu'il se donne l'espace pour le réinterpréter hors du temps, comme pure origine, comme Modèle inengendré et détaché. Raconter l'arkhè première, c'est donc accepter de récapituler dans une physique limitée ce que les présocratiques, selon des projets différents, avaient pensé des causes de la nature et de l'être, mais c'est aussi bien soumettre cette physique à un récit qui la déborde et qui seul possède le pouvoir de produire la raison du monde, sa cause véritable, dût-elle se déployer à travers le mythe d'un reflet prismatique transcendant.

Cette double dimension demande qu'on s'y arrête un peu, en raison de son importance dans la détermination grecque de la métaphysique. Si en effet le pouvoir explicatif de l'origine comme commencement est si puissant dans les théogonies et dans les cosmogonies présocratiques, c'est parce que la généalogie y commande de l'extérieur l'essence même de l'attente de pensée. Comment pourrait-on ne pas accéder à une explication parfaite, si cette explication ouvre le chemin de l'origine? Cette présupposition repose en effet sur plusieurs aspects qui mettront du temps à s'expliciter dans la pensée postérieure, en particulier dans le néoplatonisme. La notion même du principe exprime cette présupposition de manière transparente, puisque, aussi bien en grec que plus tard dans la langue latine, le principe est commencement et commandement: l'arkhè est le point de départ et la source aussi bien que le pouvoir et la détermination, elle est le commandement archontique du devenir. Le principe est aussi bien, comme le montre la langue latine qui traduit le double sens de commandement et d'origine de l'arkhè grecque, princeps que principium. Ainsi s'expose le fait que l'accès à l'origine est toujours déjà accès au réservoir infini de ce qui commande le déploiement de la suite, à la source énergétique du procès. Ces aspects, faut-il le dire, sont inconnus dans des pensées comme celles de la Chine, où le procès se structure à compter d'une tension dans une polarité et se passe entièrement du pouvoir et $d u$ commandement d'une origine?

Cette double dimension impose également un regard particulier sur les conséquences de cette recherche de l'arkhè pour celui qui l'entreprend. Le poète, le théologien, le phusikos et ultimement le philosophos, tous se placent dans la situation critique de conquérir une position de parole sur l'inaccessible, sur l'imprononçable ${ }^{8}$. Le travail de la discussion rationnelle ne saurait certes demeurer prisonnier des premières inspirations de la poésie, mais il ne perd jamais la conscience du lien profond qui l'unit à elle. Le sens de l'invocation platonicienne, que nous retrouverons plus tard dans les prières de Proclus, est précisément de reconnaître la soumission de la parole philosophique sur l'origine au discours muet de ceuxlà seuls qui pourraient, en toute justice et vérité, la 
proférer: les dieux, les Muses ${ }^{9}$. L'effort philosophique dans la tradition platonicienne ne se sépare jamais de cette humilité et en plusieurs endroits des dialogues où on attendrait que la raison affirme sa percée, on assiste plutôt à un retrait, à un repli hors du langage et dans plusieurs cas à l'investissement du langage de l'autre, c'est-à-dire de la parole du mythe dont l'essence est de se déployer à compter d'une origine non humaine. Comment prétendre en effet accéder dans le temps et dans l'histoire à ce monde qui est atemporel et anhistorique? La philosophie platonicienne, et cela sera constant dans toute sa tradition, n'est pas d'abord la recherche rationnelle d'une captation de l'origine, mais le projet, par le moyen de cette recherche, d'un retour de l'âme à sa source. Son projet de theôria contemplative déborde d'emblée celui d'une physique et même le Timée peut être considéré comme un exercice spirituel de retour vers ce monde séparé autant que comme l'effort d'une physique des causes ${ }^{10}$. Plusieurs gnoses tardives, qui se sont inspirées de cette position platonicienne, pour ne rien dire de la théologie chrétienne qui a réinterprété pour elle-même ce drame platonicien du retour à l'origine, se sont montrées à certains égards plus proches du projet de Platon que les commentaires purement rationalistes sur la séparation et le dualisme qui en ont été produits par la suite dans l'histoire de l'ontologie.

Malgré ces difficultés, qui sont les apories mêmes de la raison grecque faisant son chemin vers la métaphysique et découvrant dans la pensée de Platon la distinction de l'être et de l'étant dans la figure même de la séparation et de la différence, l'origine a été intégrée dans l'ensemble complexe des concepts de la causalité. Il serait difficile d'affirmer que c'est le concept de l'origine qui a provoqué seul la structuration de la cause, car on pourrait du même coup faire valoir que plus la métaphysique a progressé, plus elle s'est détachée de la pensée de l'origine pour adopter un langage moins chargé d'implications aporétiques dans la représentation du temps. La généalogie se trouvait déjà purifiée dans l'abstraction du concept de genèse, c'est-à-dire du pur devenir de la nature et ce concept fut à son tour emporté dans une polarité avec le concept d'être qui déjà chez Parménide isolait le devenir de la naissance. C'est le cas, par exemple, de toutes les structures sémiotiques au sein desquelles la cause se reflète dans ses effets et, en général, de toutes les doctrines de la représentation que Platon a abondamment développées, en se fondant sur ce qu'il recevait de la distinction présocratique de l'être et de l'apparence. Ce rapport instaurateur du modèle et de la copie, du réel et du simulacre, pouvait-il se passer de l'origine? S'en passet-il même chez Platon? Une étude qui se limiterait à la métaphysique de la République pourrait être tentée de conclure que cette métaphysique est exempte de toute dimension généalogique et que l'origine y a été absorbée entièrement par le modèle éternel des Formes séparées. Un pur rapport de symétrie rapporterait le monde à son archétype éternel, sans qu'il soit besoin d'introduire le récit d'un surgissement ou d'une provenance, encore moins celui de l'origine même de la différence. Le rapport de l'éternité au temps, en son point limite, excède la représentation du reflet: la dépendance en constitue certes la figure fondamentale, mais cette dépendance n'est pensable dans la philosophie que si celle-ci accepte la figuration mythique de la chute. Autrement, l'événement du devenir demeure impensable et la métaphysique sera portée à conclure que le temps est une illusion. Cette conclusion est rendue nécessaire par le refus de toute perspective de création qui, en introduisant une tangence de l'éternité au temps, a toujours paru aux Grecs menacer l'essence pure de la divinité.

Mais cela serait faire trop peu de cas du texte de la République (VI, 509b), c'est-à-dire de cette prégnance du Bien qui illumine l'étant en le produisant et que Platon n'hésite pas à présenter dans l'image de la paternité. Dans ce texte, commenté dans toute la tradition platonicienne et en particulier par Plotin, la position de l'au-delà, de cet epekeina qui ouvre la transcendance, ne peut se passer de l'originarité: l'éternité ne peut certes se placer avant le temps, mais elle est la source du temps. Rien certes dans la 
République n'explique le passage au temps, c'est-à-dire l'avènement de la différence, mais l'origine demeure métaphysiquement constituée. Il faut attendre plusieurs siècles de réflexion au sein de la tradition platonicienne pour trouver, dans le traité III, 7 de Plotin sur l'éternité et le temps, la réponse la plus élaborée de la métaphysique aux apories de la doctrine de l'origine.

Cette métaphysique trouve son expression cardinale dans un passage du Timée, qu'on peut considérer comme le cœur de la doctrine platonicienne de la causalité ${ }^{11}$. Après avoir présenté ce qu'il considère comme les causes adjuvantes ou auxiliaires du mouvement, causes qui servent le grand dessein téléologique du dieu artisan-démiurge dans la production du meilleur, Platon introduit la notion d'une causalité supérieure, celle qui accède aux causes rationnelles et premières. Les causes auxiliaires ne sont en effet que des mécanismes intermédiaires de transmission, la pensée exige d'accéder à la cause originaire.

[...] Celui qui est amoureux de la raison et du savoir doit nécessairement rechercher, comme premières, les causes qui ressortissent à ce qui par nature est rationnel et comme secondes, toutes celles qui ressortissent à ce qui reçoit son mouvement d'autres êtres déjà en mouvement, et qui, en obéissant à la nécessité, transmet ce mouvement à d'autres. C'est bien ainsi qu'il nous faut procéder nous aussi. Certes nous sommes obligés de parler des deux sortes de causes, tout en distinguant bien entre toutes celles qui donées d'intelligence produisent des choses belles et bonnes, et toutes celles qui privées de raison produisent à tout coup leurs effets au hasard et sans ordre. (Timée, 46d-e) ${ }^{12}$

Ce passage s'illumine si on le met en rapport avec le texte parallèle du Phèdre, dans le grand exposé sur l'immortalité de l'âme. De la même manière en effet que le Monde, qui est phusis et histoire, ne trouvera son explication que dans une originarité qui dépasse le mécanisme, l'immortalité de l'âme ne sera pensable que si on arrive à saisir l'originarité de son mouvement propre.

Il n'y a que ce qui se meut soi-même qui, du fait qu'il ne se délaisse pas soi-même, ne finit jamais d'être en mouvement; mais en outre, il est, pour tout ce qui est encore mî, une source et un principe (arkhè) de mouvement. Or un principe est chose inengendrée, car c'est à partir d'un principe que vient à l'existence tout ce qui commence d'exister, au lieu que lui-même, nécessairement, il ne provient de rien; si en effet, il commençait d'être à partir de quelque chose, il n'y aurait pas commencement d'existence à partir d'un principe. (Phèdre, 245c-d) 13

On voit comment Platon répond à une injonction qui a toutes les allures d'un axiome de la métaphysique grecque. Une cause originaire ne peut en effet qu'être elle-même non causée, et dans l'automotricité substantielle de l'âme, principe fondamental de la pensée, la philosophie est invitée à reconnaitre le seul modèle possible d'une origine: être à soi-même sa propre origine. Tout le procès de la phusis, parce qu'il est procès et développement de causes qui le dépassent, constitue donc le déploiement d'une cause originaire, désignée comme principe (arkhè) ${ }^{14}$. L'interprétation de cet axiome exige de recourir à la fois aux images qui l'expriment et à la doctrine de la priorité de la pensée. Il s'agit en effet de deux propositions en apparence indissociables dans les nombreux textes où Platon les présente comme la limite même de la pensée de l'origine, mais on peut tenter de les distinguer. Sur un premier registre, nous sommes mis en présence d'une proposition sur la nature de la cause en tant qu'origine: une origine première, cause originaire, est nécessairement dépourvue elle-même d'origine. Cette proposition va se développer de manière forte dans la doctrine subséquente de la cause de soi, causa sui, dont Plotin va faire la proposition centrale de la théologie ${ }^{15}$. La seule définition acceptable du premier Principe, dans une doctrine qui refuse toutes les affirmations et toutes les désignations positives pour se replier sur la théologie négative, sera la causalité de soi. Nous sommes donc invités à repérer, sur le plan du déploiement du langage de l'arkhè, le prédicat de divinité comme prédicat défini par un prédicat logiquement antérieur, celui de l'être auto-causé. Mais cette proposition trouve à son tour sa justification ultime dans les affirmations du Phèdre et du Timée: si 
en effet la causalité de soi est primitive, si elle parvient à définir le divin, c'est d'abord parce qu'elle est prédiquée de la pensée, c'est-à-dire de ce qui échappe à l'ordre des corps et subsiste dans la nécessité de l'intelligible éternel. Cette deuxième proposition n'est certes pas dissociable de la première, et ceci ne se voit nulle part mieux que dans la définition du divin comme pensée et intellect, une thèse de la métaphysique aristotélicienne que toute la tradition ultérieure considérera comme le fondement même de la métaphysique.

Déjà chez Platon, et cela le texte du Sophiste l'affirme clairement, la Nature comme phusis se produit selon le procès d'une cause originaire. En affirmant que tous les êtres, animés et inanimés, sont engendrés par un dieu démiurgique (Sophiste, 265c4), Platon reprend la doctrine du Timée, qu'il se hâte de désigner comme langage philosophique échappant aux apories du langage vulgaire, le langage commun. De quelle conception veut-il ici se dégager, sinon celle d'une causalité neutre ou purement mécanique, qui tout en invoquant le procès des causes, s'interdit d'y voir le travail d'une pensée? Selon cette conception ordinaire, la nature engendre les êtres «selon une cause automatique, spontanée, qui se développe sans le secours d'aucune pensée " ${ }^{16}$. La menace d'un arbitraire mécanique suffit à disqualifier un concept de cause originaire privé de sa détermination divine. Cela, Platon l'affirmait également dans le Philèbe, quand il plaçait la cause originaire non pas du côté d'une force irrationnelle, procédant au hasard et arbitrairement, mais selon l'ordre de la Pensée, selon la Raison admirable (Philèbe, 28c). Impossible donc de dissocier, dans la doctrine de l'origine, l'assignation de son concept pur d'auto-causalité, source du concept théologique de la cause de soi, d'une métaphysique de la Pensée divine, c'est-à-dire de l'origine en tant que prédicat limite de la divinité.

Dans cette affirmation, nous ne pouvons que constater à quel point les mythes archaïques de Chronos, les généalogies hésiodiques et l'ensemble du mythe du temps sont à la fois intégrés et transformés par la métaphysique. Le pouvoir de l'origine mythique, qui désigne d'emblée un en-deçà du temps et de l'Histoire, est d'en commander le déploiement et d'en régir, d'une position transcendante, les événements et le devenir corruptible. La doublure du monde est à la fois son origine et sa cause actuelle, ce qui légitime aussi bien la nostalgie des âges héroïques que la prière, le souhait de voir intervenir l'origine dans le présent et le désir, en récapitulant le destin de l'âme, de retourner par la philosophie à l'origine. Si Platon refuse un mécanisme aveugle, auquel il associe certainement plusieurs doctrines présocratiques, c'est dans un geste par lequel il maintient l'arkhè divine non seulement comme origine, mais comme pouvoir sur le monde. La pensée qu'il évoque, son dessein rationnel, la grandeur de l'ordre auquel ce logos préside, hérite en son entier des prédicats de la divinité hésiodique. À l'arbitraire aveugle d'un Anaxagore, que Socrate dénonce dans le Phédon (96a), il substitue donc la présence éternelle d'une cause parfaite dont les lois de la Nature, aussi bien que les mouvements de l'âme, sont l'expression dans le temps. Mais ces lois et ces mouvements n'en sont que l'expression, car, en eux-mêmes, ils ne sont pas la Pensée. Cette séparation constitue certes l'abîme du platonisme, un écart aussi mystérieux qu'insondable qu'aucune image du reflet ou de la copie n'arrive à franchir de manière satisfaisante.

Dans le Sophiste (257c), Platon affirme bien que la nature de l'autre brille en éclats dispersés dans l'étant, comme un savoir. Cette brillance morcelée de l'origine n'apparaîtrait pas si elle n'était de la même nature que l'âme, et, par conséquent, le khôrismos de la séparation originaire semble franchissable, mais dans le moment même où il le laisse espérer, Platon n'a de cesse de durcir pour la crisper la distinction de l'être et de l'étant qui est la limite de sa métaphysique. Ce que l'originarité de l'âme laisse entrevoir, c'est la possibilité d'un accès à l'origine; ce que la séparation de la différence interdit, c'est la certitude pour la philosophie que cet accès lui soit consenti dans les seules limites du travail de la pensée. Ici cependant s'ouvre un autre volet, celui du dépassement de la pensée, de la transcendance de l'âme non seulement 
dans son mythe de retour à l'origine, un récit qui encadre toute la métaphysique, mais dans l'irruption de l'amour qui exauce son désir le plus profond, celui d'échapper au temps.

\section{Provenance ET ATTACHEMENT}

Dans la pensée postérieure à Platon, une pensée qui va instituer le Timée comme modèle déterminant de la métaphysique du temps, l'expression de la relation d'image va s'imposer avec force, mais elle s'associera avec l'expression de la dépendance et de la continuité qui trouve son achèvement dans le néoplatonisme. Impossible en effet de penser le temps sans le rapporter à son modèle qui est l'éternité, et ce rapport est le rapport d'une image à son archétype (Timée, 37a). Mais cette impossibilité commandait un effort pour rétablir une continuité au-delà de la brèche introduite par le khôrismos. C'est de là que Plotin va vouloir reprendre la question de l'origine, dans une entreprise qui cherche une prise directe et contemplative à compter de la similitude, de cette homoiotès qui fait du temps le ciel entier et l'événement du monde, alors que l'éternité est un être sublime (semnos, Ennéades, III, 7,2,5) auquel ce monde ressemble. La question lancinante de Plotin demeure cependant celle de la présence de cette sublimité éternelle dans le temps de la similitude, puisque l'éternité, comme la nature intelligible, contient le temps. Cette expression est certes une image impure, mais elle désigne aussi bien l'environnement que la présence. Et nous nous retrouvons ici, comme le plus souvent dans le texte plotinien, devant un effort extrême pour purifier le langage, un effort qui ne trouve son aboutissement que dans le repli sur la relation pure de la différence, c'est-à-dire dans une expression dynamique sublimée. Être dedans, être autour, dépendre de, provenir, se rattacher, surgir de constituent en effet autant d'efforts de réduire les scories du langage de la métaphysique pour se concentrer sur l'expression pure.

«L'éternité est un tout qui ne se partage pas» est à cet égard une proposition aussi nécessaire qu'impensable, car sa nécessité lui vient de son concept, alors que son impossibilité découle de la pensée de la nature intelligible. Ne faut-il pas en effet trouver dans l'éternité, dans sa contenance même, l'origine non seulement du devenir temporel, ce que déjà le Timée avait proposé, mais des substances éternelles de la nature intelligible, c'est-à-dire, dans le lexique de Plotin, de l'Intellect contenant les Formes? Le traité III, 7 des Ennéades introduit à cet égard une récapitulation complète des concepts du Sophiste, et en particulier de l'identité et du repos dans l'être. Mais il va beaucoup plus loin, dans la mesure où il déporte l'éternité dans un au-delà substantiel, dont participent les êtres intelligibles, mais qui est lui-même un originaire qui n'est pas. L'origine est au-delà de ce qui est, c'est ainsi que Plotin comprend la thèse de République VI, 509b et il en tire les conséquences de la manière la plus radicale. Pour quelle raison? L'enjeu métaphysique est pour lui déterminant: comme la nature intelligible contient la multiplicité des Formes éternelles constitutives de l'Intellect, l'éternité est une austostasis qui le transcende, un repos en soi. C'est une vie qui persiste dans son identité, au rang même de l'originarité du Principe Un.

Aussi l'éternité est-elle non pas le substrat des intelligibles, mais en quelque sorte le rayonnement qui en provient, grâce à cette identité que le principe affirme de lui-même, non pas avec ce qu'il sera plus tard, mais avec ce qu'il est. Il est ce qu'il est et ne sera pas autrement. Que lui adviendraitil qu'il ne soit dès maintenant? (III, 7,3,16)

La nature divine de cette éternité appartient aussi à sa description métaphysique et Plotin s'empresse d'en placer le concept dans la proximité de l'Un: l'éternité est une vie totale qui est auprès de l'Un: «[...] elle vient de lui et va vers lui; elle ne s'en va pas loin de lui, mais elle reste toujours près de lui et en lui, et elle conforme sa vie sur lui ». Ce passage est présenté dans un rapport au texte de Platon (Timée, 37e) qui en constitue certes le foyer, mais Plotin lui apporte une interprétation radicale. C'est en effet la contraction de l'origine dans l'Un, au sein de cette éternelle présence de soi à soi, qui exprime le concept le plus pur et le plus élevé de la métaphysique de l'origine. Tout ce qui en dérive et en 
découle n'en est que la dilatation ou la diffraction dans des registres ontologiques inférieurs, qu'il s'agisse de l'Intellect ou de l'Âme. De cet écoulement, de ce rayonnement, où la tradition a reconnu la métaphore centrale du néoplatonisme, on peut tirer certes le principe d'une continuité susceptible en son principe de rétablir le rapport brisé de la différence, de vaincre le khôrismos platonicien, mais on ne peut éviter d'y voir en même temps l'expression d'une perte et d'un éloignement continus, qui atteignent, avec la matière, le seuil du néant.

Plotin construit en effet un double effort: d'une part, il concentre dans un Premier, qui n'est pas, parce qu'il est au-delà de l'être même des natures éternelles et intelligibles, la présence de ce qui est par soi, en soi, auprès de soi, tourné et incliné vers soi-même. Le langage de la métaphysique se porte ainsi aux limites de ces mouvements qui font de la prépositionnalité la dynamique la plus sublime du langage, son fond le plus abyssal. Mais dans le moment même où cette contraction des mouvements de la pensée qui cherchent le centre élabore le point limite de cette origine de vie qui est la présence, Plotin accepte d'en inverser le mouvement en direction de l'être et d'instaurer ce surgissement qui s'écoule, cette source qui irrigue, ces rayons qui en illuminant font être et connaître, cette éternité qui en se diffusant produit le temps qui la contemple et s'abîme de désir dans la joie de la retrouver. Une nouvelle structure de mouvements s'élabore, qui est l'inverse de la première: non pas vers, mais en provenance de (ek, apo), non pas auprès de, mais à partir de et dans la dépendance de, et ainsi s'instaure la métaphore centrale de la procession (proodos), qui est le récit de la dépendance de tout être par rapport à son origine. Proclus, lui aussi grand commentateur du Timée, fera de ce concept le cœur de sa métaphysique.

Ce langage concentré des prépositions de la métaphysique avait été enclenché dans les générations précédentes des interprètes de Platon, chez ces penseurs qui étaient les préparateurs du néoplatonisme, pour reprendre l'expression de W. Theiler ${ }^{17}$. Le moyen platonisme avait en effet construit tout un édifice métaphysique en multipliant les rapports à l'origine, pour tenter de circonscrire la dépendance de l'origine dans un vocabulaire qui dépasse celui de la physique. On a parlé pour désigner leur effort de «transcription prépositionnelle» de la métaphysique ${ }^{18}$, et il s'agit de fait d'un travail sur la série platonicienne de la dépendance des principes: à partir de quoi, en dépendance de qui, en retournant vers qui. Dans les premiers temps de cette tradition, ce langage fut reçu comme une entrave à la pensée des principes, mais Plotin et Porphyre eurent tôt fait de reconnaitre chez leurs prédécesseurs médio-platoniciens une recherche qui conduisait à leur projet. Une recherche qui établit un ordre d'instances intelligibles et introduit entre elles une relation de surgissement ne pouvait en effet qu'inspirer le néoplatonisme. Si l'écoulement de la source semble une image trop sensible, si sa poétique est trop matérielle, on retranchera la substance de l'expression pour ne conserver que la préposition: non pas aporreon ek, mais seulement $e k$, produisant de la sorte un langage métaphysique de l'origine entièrement purifié de tout ce qui n'est pas le pur mouvement de la différence. L'origine s'exprime dès lors dans une relation pure.

Déjà dans le traité III, 2 sur la providence, où Plotin reprend pour les critiquer les concepts stoïciens de la semence, on voit l'effort néoplatonicien pour isoler la pure puissance nécessaire de ce qui se développe dans l'être (III, 2, 2, 17-31). Les récits gnostiques, tout comme les reprises par Philon d'Alexandrie des formules de la création, ont beaucoup contribué à faire de ces séries de rapports une sorte de vulgate de la métaphysique platonicienne des deux premiers siècles de notre ère. Certains interprètes la désignent même comme stoïcognostique, ce qui laisse supposer une intime interpénétration des conceptions métaphysiques de la période impériale. On en retrouve par exemple l'expression chez Sénèque (Lettre 65,11), lorsqu'il identifie la recherche de la cause originaire à la série des rapports de provenance et de point de retour: de quoi, à travers quoi et vers quoi. Cette série trouve peutêtre son origine dans la religiosité orphique pré- 
hellénistique, mais elle s'est intégrée dans la métaphysique de l'origine sur le terrain de l'exégèse du Timée, où elle va s'implanter jusqu'au commentaire tardif de Proclus. On peut citer par exemple le passage de Marc Aurèle, où nous la retrouvons:

Tout est fruit pour moi de ce que produisent tes saisons, $\hat{o}$

Nature! Tout vient de toi, tout est en toi, tout rentre en toi.

(Pensées pour moi-même, 4, 23)

Ce texte parait faire écho, en sa structure même, au texte de l'Épître de Paul aux Romains, $(11,36)$, comme à tant d'autres textes de cette période de diffusion du platonisme dans la culture impériale ${ }^{19}$. Mais son intérêt réside surtout dans le fait que cette structure prépositionnelle de l'ontologie semble avoir été la solution de la tradition platonicienne pour dépasser la séparation de la pure différence, et laisser entrevoir non seulement l'espoir d'un retour à l'origine, mais la possibilité d'un récit d'avènement et la continuité d'une histoire qui s'achève dans une eschatologie. On tirerait sans doute dans une direction extrême le texte de Plotin si on tentait de le réduire à ce récit. Plotin a tenu avec rigueur à la position d'un Un radical et originaire, qui s'accomplit dans son acte, qui est toujours le même et ne diffère jamais de lui-même. De sorte que le procès par lequel les êtres intelligibles procèdent de lui demeure éternellement suspendu à la question de sa différenciation dans une altérité. Les traités V, 1 et VI, 7 sont à cet égard les témoins importants de l'effort plotinien pour introduire non pas le mouvement dans l'Un, mais pour raccorder de la manière la plus extrême l'origine et la différence. Plotin crut pouvoir en trouver l'expression dans une epistrophè, un retournement vers soi qui serait productif d'un autre. Thèse radicale s'il en est, cette proposition engage la suite de la procession des êtres, qui tous seront soumis à cette conversion vers le principe, seule garante de leur identité et de leur rapport à l'origine.

C'est ainsi que Plotin croit possible de demeurer fidèle à la doctrine du Timée. L'Intellect éternel, luimême dérivé par epistrophè de l'Un, n'existe pas avant le monde, mais il est le monde avant son être:
Grâce à ce qui est au-dessus de lui, le monde n'existe pas à partir d'un certain moment; et ainsi le monde ne peut avoir eu un commencement dans le temps. C'est le seul fait d'être cause qui donne à l'être son antériorité. (III, 7, 6, 52-54)

Comment comprendre une telle antériorité non temporelle? Est-elle purement logique? Il convient sans doute de tenter de la penser sur le fond, déjà perceptible chez Platon, d'une éternité du monde sensible accompagnant dans une dépendance infinie l'éternité non temporelle. Ce point limite est celui qu'a cherché à exprimer Plotin dans l'extraordinaire récit du traité III, 7, alors qu'il entreprend de raconter l'avènement du temps comme provenance, attachement à un registre dont l'être a chuté. «De quelle chute, demande-t-il, est donc né le temps?». La réponse est une admirable prosopopée du temps, qui invoque le désir de la nature, inquiète et curieuse de son propre devenir, de se porter au-delà de son propre accomplissement pour le contempler. Ce geste fut celui d'une entité prodigue d'elle-même, dispensatrice de sa force vers une extériorité en mouvement: dans ce geste de générosité, la nature se fit elle-même temps et elle soumit au temps le monde engendré par elle. Ce récit, qui mériterait une longue exégèse, en particulier parce qu'il se veut une interprétation non démiurgique du Timée, incorpore un concept pur de Nature, emprunté en plusieurs points, quelles qu'aient été ailleurs les réserves de Plotin à leur endroit, aux doctrines stoïciennes de la germinalité. C'est ce récit qui conduit Plotin à identifier l'âme au démiurge platonicien, ce qui a pour lui deux conséquences essentielles: quitter le dispositif artisanal, et surtout relier l'âme au monde dans un rapport de pure dépendance continue.

Dans le contexte de cette réinterprétation du Timée, Plotin, comme plus tard Proclus, réinvestit entièrement la série des principes du rattachement: si l'âme est la première, pour le monde comme pour les individus, à aller vers le temps, en l'engendrant pour le posséder avec ses propres actes, c'est que l'âme dilate pour ainsi dire l'éternité dans le temps. Partout où il y a de l'âme, il y a du temps, ce qui ne signifie 
aucunement que l'âme soit matérielle, mais seulement qu'elle se dispose dans une distance d'avec l'éternité qui marque un écart ontologique et une différence. Cet écart n'induit aucune séparation, seulement une sorte d'éloignement, qui inspire la nostalgie de l'origine et stimule le désir du retour. Toute la tradition ultérieure, et on pense ici à saint Augustin, sera pénétrée de cette présence quasi réelle de l'origine dans le temps, même si la structure de la métaphysique chrétienne, parce qu'elle incorpore la doctrine de la création, se place en rupture complète avec la pensée grecque. Même si l'Un plotinien n'est pas la pensée, même s'il n'est pas en tant que tel, puisqu'il est au-delà de l'être, on ne peut l'identifier à un pur néant originaire. Son concept ne se réduit pas à sa seule fonction métaphysique d'engendrement par retournement de ce qui le suit éternellement. Plotin a cherché avant tout à dégager son Principe de toute détermination purement intelligible qui le rapprocherait trop du Dieu aristotélicien, Pensée de la Pensée, et quand il accepte de le déterminer positivement, il le désigne comme veille, liberté, pure position de soi-même. C'est la leçon du traité VI, 8, le grand texte où les consignes de la théologie négative paraissent débordées par un langage qui ne recule pas devant la positivité de désignations de l'Un comme liberté et volonté.

Il est vrai que le néoplatonisme médiéval et renaissant - pensons seulement à l'œuvre de Nicolas de Cues - accentuera fortement cette affirmation du néant comme non-être, mais en cela il ne sera fidèle qu'au désir de Plotin de protéger l'Un de toute détermination dangereuse pour sa sublimité. Quand Plotin en effet écrit que «[...] c'est parce qu'il n'y a rien dans l'Un que toutes choses proviennent de Lui: afin que l'être soit, il faut que Lui-même ne soit pas être, mais le générateur de l'être» $(\mathrm{V}, 2,1,11)$, il ouvre la porte à toutes les mystiques négatives qui acceptent la séparation de l'Un et de l'être et consentent à se replier sur une négativité entière pour penser l'origine. Être l'origine, c'est par nécessité n'être rien, car autrement on serait déjà le sujet de déterminations particulières, et le statut de l'origine ne serait plus celui d'une origine authentique. Cet héritage de pensée sera recueilli par Proclus et Damascius, qui sont les maîtres du néoplatonisme médiéval d'Eckhart et du Cusain. Leur commentaire du Parménide a porté, sur le registre du langage pur de la négativité, ce qui s'était transmis jusqu'à eux, et notamment par l'œuvre de Porphyre, de la doctrine platonicienne de la différence ${ }^{20}$. Ce langage est certes bien différent de la démiurgie du Timée, que Plotin avait réinterprétée dans son concept d'âme; il n'est pas substantiellement différent cependant de cette recherche incessante de penser l'origine dans une présence au temps qui ne soit pas une présence saisissable, mais un acte éternellement co-existant à sa radiation mystérieuse. Cette leçon du néoplatonisme, Platon ne l'aurait peutêtre pas reconnue, lui qui dans la recherche de l'arkhè première avait d'abord isolé une cause séparée, mais il aurait néanmoins entendu le langage du désir de l'âme de retrouver sa patrie négative, cette origine sans déterminations que déjà dans le Parménide il avait offerte à la spéculation de la tradition.

\section{NO TES}

1. Dans une étude d'une grande richesse, inspirée par le désir de retracer la généalogie du «Au commencement» du prologue de l'évangile de Jean, A. Ehrhardt propose une remontée à travers toute l'histoire de la pensée grecque. Voir son livre, The Beginning. A Study in the Greek Philosophical Approach to the Concept of Creation from Anaximander to St John, Manchester, Manchester University Press, 1968, 212 p. Il est certes risqué de produire pour le monde grec une transcription de l'origine dans le concept de création, mais cette étude comparative a le mérite de mettre en relief tout ce qui dans la pensée grecque résiste à la position de la création.

2. Voir l'étude de G. Naddaf, L'Origine et l'évolution du concept grec de phusis, Lewiston (Maine), The Edwin Mellen Press, 1992, 603 p. Ce

livre montre la continuité de l'enquête sur la nature, des présocratiques au Timée et aux Lois de Platon.

3. M. Richir a fait de cette analogie le thème d'une réflexion sur la limite théologique de l'assignation de l'origine, voir La Naissance des dieux, Paris, Hachette, coll. "Essais du XXe siècle", 1995, 191 p.

4. Dans la cosmologie présocratique, ce texte est le plus important. Voir l'édition et le commentaire de M. Conche, Anaximandre. Fragments et témoignages. Texte grec, traduction, introduction et commentaire, Paris, P.U.F., coll. "Épiméthée», 1991, 245 p.; en particulier les pages 55-62, sur l'arkhè. M. Heidegger a consacré aux fragments d'Anaximandre un 
essai devenu célèbre, «La parole d'Anaximandre», dans Chemins qui ne mènent nulle part, Paris, Gallimard, coll. "Classiques de la philosophie", 1962, p. 262-304. Dans cet essai, il insiste sur le rapport de l'origine comme présence dans le séjour de l'étant: «Plus secrètement encore s'y recèle: présence elle-même comporte venue au jour. La venue au jour de l'éclosion elle-même est présence». Voir sur l'ensemble des études de Heidegger sur les présocratiques le livre de M. Zarader, Heidegger et les paroles de l'origine, préf. de E. Lévinas, Paris, Librairie philosophique J. Vrin, 1986, 319 p.

5. Fragments 2 et 3, selon la numérotation de l'éd. de M. Conche, op. cit., p. 56-57. La doxographie et les fragments d'Anaximandre sont recueillis dans la coll. de H. Diels, s. la dir. de W. Kranz, Die Fragmente der Vorsokratiker, Berlin, Weidmann, 6e éd., 1951; voir vol. II, p. 81-90, avec la numérotation des passages doxographiques et des fragments (DK 12).

6. Voir à cet égard le livre de S. Margel, Le Tombeau du dieu artisan, préf. de J. Derrida ( Avances»), Paris, Éd. de Minuit, 1995, 204 p.

7. Voir à cet égard les travaux de F. Jullien, un des rares interprètes à tenter une interprétation comparative de la pensée grecque et de la pensée orientale. Notamment Procès ou création. Une introduction à la pensée des lettrés chinois, Paris, Seuil, coll. «Des travaux», 1989, en particulier le chap. 5 qui introduit une comparaison avec le Timée de Platon et la critique de la cause première.

8. M. Loreau, dans un essai important, a cherché à montrer comment cette conquête doit rompre avec la tradition platonicienne, qui n'est que la construction d'un mythe de l'origine; mythe décisif, mythe nécessaire sans doute, mais encore et toujours mythe. Voir « La philosophie comme construction nécessaire du mythe d'origine», dans Le Temps de la réflexion, Paris, Gallimard, vol. I, 1980, p. 315-339.

9. R. Brague a consacré à ces conditions du discours du commencement une étude, «Le récit du commencement. Une aporie de la raison grecque", dans J.-F. Mattéi, La Naissance de la raison en Grèce, Paris, P.U.F., 1990, p. 23-32.

10. C'est ce que soutient P. Hadot, dans son article «Physique et poésie dans le Timée de Platon ", Revue de théologie et de philosophie, 115 (1983), p. 113-133.
11. Le commentaire contemporain du Timée est dominé par les travaux de L. Brisson, voir Le Même et l'Autre dans la structure ontologique du Timée de Platon, Paris, Klincksieck, coll. «Études et commentaires», 1974, 589 p., auquel il faut joindre un essai qui cherche à développer une perspective comparative avec la physique contemporaine, en coll. avec W. Meyerstein, Inventer l'univers. Le problème de la connaissance et les modèles cosmologiques, Paris, Les Belles-Lettres, coll. «Lâne d'or ", 1991, $209 \mathrm{p}$.

12. Trad. de L. Brisson, Paris, Flammarion, 1992, légèrement modifiée.

13. Trad. de L. Robin. Platon, Euvres complètes, Paris, Gallimard, coll. "La Pléiade», 1950.

14. Plusieurs passages platoniciens reviennent sur cet axiome, notamment le Philèbe (26e) et le Timée (28a). Dans le Sophiste (265b), c'est tout le domaine de la poiêsis qui est soumis au procès de la cause, et de même dans le Banquet (205b): pour que le non-être prenne le chemin de l'être, il faut une cause originaire.

15. L'histoire de ce concept est déterminante, puisque la limite de l'origine est le concept de ce qui est à soi-même sa propre origine. Voir l'étude de J.-M. Narbonne, La Métaphysique de Plotin, Paris, Librairie philosophique J. Vrin, coll. «Bibliothèque d'histoire de la philosophie", 1994, p. 26ss.

16. Sophiste, 265c8-9, trad. d'A. Diès, Paris, Les Belles Lettres, coll. des Universités de France, 1925.

17. W. Theiler, Die Vorbereitung des Neuplatonismus, Berlin, Weidmann, 1964.

18. Voir par exemple l'étude de H. Dörrie, «Präpositionen und Metaphysik. Wechselwirkung zweier Prinzipienreihen", Museum Helveticum, XXVI, 1969, p. 217-228.

19. Dörrie cite par exemple le traité Asclepius du Corpus Hermeticum (éd. Festugière-Nock, II, 344, 26) : « [...] omnia enim ab eo, et in ipso et per ipsum ». Et encore Plutarque, De Animae procreatione, 5, 1014ab, qui reprend précisément le texte du Timée; voir H. Dörrie, op. cit., p. 222. 20. Voir l'étude d'É. Bréhier, «L'idée du néant et le problème de l'origine radicale dans le néoplatonisme grec", dans Études de philosophie antique, Paris, P.U.F., 1955, p.248-283. 